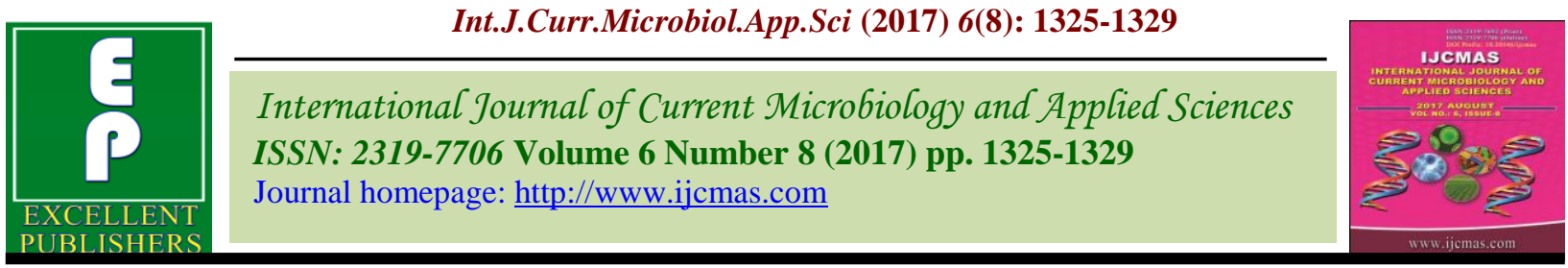

Original Research Article

https://doi.org/10.20546/ijcmas.2017.608.161

\title{
Tube Adherence Test as a Screening Tool for Detection of Biofilm Formation among Staphylococcus aureus
}

\author{
K. Ram Mohan Reddy* \\ Rajiv Gandhi Institute of Public Health and Center for Disease Control, 426/27, 33rd Cross, \\ 18th Main, 4th T Block, Jayanagar, Bangalore -560 041, India \\ *Corresponding author:
}

\section{A B S T R A C T}

Keywords

Biofilm,

Staphylococcus

aureus,

Tube method, Specificity.

Article Info

Accepted:

17 June 2017

Available Online:

10 August 2017
In view of large number of infections caused by biofilm forming organism, a reliable method for its detection is necessary. Highly accurate methods like Polymerase Chain Reaction (PCR) are available for detection of biofilm, but these are beyond the scope of majority of microbiology laboratories in developing nations like India. To test reliability of Tube Adherence test as a screening tool for detection of biofilm formation among Staphylococcus aureus. With universal safety precautions various clinical samples were collected from patients with indwelling medical device for more than 48 hours. Screening for biofilm detection was done using Tube adherence method and tissue culture plate method. Tube method was $97.5 \%$ sensitive and $100 \%$ specific for detection of biofilm formation. Tube method can be used as a general screening method for detection of biofilm producing bacteria in laboratories.

\section{Introduction}

Biofilm production is an important factor in most of the nosocomial infections. The increased use of indwelling medical devices has had considerable impact on the role of Staphylococci in clinical medicine (Mathur et al., 2006). Staphylococci are most often associated with chronic infections of implemented medical devices. Staphylococci are recognized as the most frequent causes of biofilm-associated infections (Robert et al., 2014). This exceptional status among biofilmassociated pathogens is due to the fact that Staphylococci are frequent commensal bacteria on the human skin and mucous surfaces. Thus, Staphylococci are among the most likely germs to infect any medical device that penetrates those surfaces, such as when being inserted during surgery.

The differentiation of Staphylococci with respect to its biofilm phenotype might help to elucidate the impact of Staphylococci in the diagnosis of infections related to biomedical devices and these observations may have utility in the prevention of device related infections. In infection by biofilm producing Staphylococci, the differentiation with respect to biofilm phenotype might help to modify the antibiotic therapy and to prevent infection related to biomedical devices. A suitable and reproducible method is necessary for screening of biofilm producers in any health 
care setting (Oliveira et al., 2010). In this study an attempt was made to test Tube adherence test as a screening tool for detection of biofilm formation among Staphylococcus.

\section{Materials and Methods}

A total of 100 non-repetitive, clinical strains of Staphylococcus were isolated from various clinical samples of the indoor and outdoor patients of Vijayanagar Institute of Medical Sciences (VIMS), Bellary, India during one year period from January 2013 to December 2013.

With universal safety precautions various clinical samples such as urine, pus, blood, sputum, were collected from in-patients and out-patients, and were processed according to CLSI guidelines. Clinical samples like pus, blood, sputum, body fluids, corneal scrapping, and indwelling urinary catheter were collected from patients of both sexes admitted to various departments of VIMS.

Consent was taken from all patients after explaining them briefly about the study.

\section{Inclusion criteria}

All isolates of Staphylococcus spp from patients hospitalized for more than 48 hours and positive for enzyme coagulase by tube coagulase test.

\section{Exclusion criteria}

All Staphylococcus spp isolated from outpatients, with in-dwelling medical devices, but were negative for enzyme coagulase.

All out-patients without in-dwelling medical devices

Patients hospitalized for less than 48 hours.
Tube adherence test (Freeman et al., 1989) (TM)

The suspensions of the tested strains were incubated in glass tubes which contained Brain Heart Infusion Broth aerobically at a temperature of $35^{\circ} \mathrm{C}$ for a period of 2 days. Then, the supernatants were discarded and the glass tubes were stained with a $0.1 \%$ safranine, washed with distilled water 3 times and dried. A positive result was defined as the presence of a layer of the stained material which adhered to the inner wall of the tubes. The exclusive observation of a stained ring at the liquid-air interface was considered as negative. Experiments were done in triplicate for 3 times and read as absent, weak, moderate and strong.

\section{Tissue Culture Plate Method (TCP)}

Isolates from fresh agar plates were inoculated in Brain Heart Infusion broths with $2 \%$ sucrose and incubated for 24 hours at $37^{\circ} \mathrm{C}$ in stationary condition and diluted 1 in 100 with fresh medium.

Individual wells of sterile, polystyrene, 96 wells-flat bottom tissue culture plates wells were filled with $0,2 \mathrm{ml}$ of the diluted cultures and only broth served as control to check sterility and non-specific binding of media.

The tissue culture plates were incubated for 24 hours at room temperature. After incubation content of each well was gently removed by tapping the plate.

The wells were washed four times with $0.2 \mathrm{~mL}$ of phosphate buffer saline (PBS pH 7.2) to remove free-floating 'planktonic' bacteria. Biofilms formed by adherent 'sessile' organism in plate were fixed with sodium acetate (2\%) and stained with safranine $(0.1 \%)$. 
Excess stain was rinsed off by through washing with deionized water and plates were kept for drying. Adherent Staphyloccal cells usually formed biofilm on all side wells and were uniformly stained with safranine.

Optical densities (OD) of stained adherent bacteria were determined with an ELISA reader at wavelength of $570 \mathrm{~nm}$. These OD values were considered as an index of bacteria adhering to surface and forming biofilms.

Experiment was performed in triplicate and repeated three times, the data was then averaged. To compensate for background absorbance, OD readings from sterile medium, fixative and dye were averaged and subtracted from all test values. The mean OD value obtained from media control well was deducted from all the test OD values.

Classification of bacterial adherence was based on OD values. When the mean OD values were $<0.120$ it was classified non/weak biofilm formation, OD $570 \quad 0.120-0.240$ was classified moderate biofilm formation, $\mathrm{OD}_{570}>0.240$ was classified strong biofilm formation.

Experiments were performed in triplicate and were repeated 3 times, the data were then averaged. All tubes which showed layer of stained material ( safranine) to the inner walls of the tube, as seen in the tube on right of the picture above, was considered positive for biofilm formation by Tube method. All tubes with stained ring only at the liquid-air interface and not staining inner walls of the tube, as seen in the tube on left of the picture above, was considered negative for biofilm formation by Tube method.

\section{Statistical analysis}

Statistical analysis was done by conisdering the percentages and simple ratios.

\section{Results and Discussion}

Of the 100 staphylococcus samples collected, 57(57\%) were Staphylococcus aureus. Out of the 57 isolates tested for biofilm by tube method, 07 (12.28\%) were strongly positive. However maximum number of isolates 29 $(50.88 \%)$ were moderately positive and 21 $(36.84 \%)$ did not show any biofilm formation.

In the study by Fatima Khan et al., (2011) done for the detection of biofilm formation in Staphylococcus aureus using Tube method out of 262 isolates, 35(13.36\%) were strongly positive. Maximum isolates were moderately positive; $132(50.38 \%$ ) and $95(36.26 \%)$ did not show any biofilm formation. Even in our study $12.28 \%$ of the S.aureus isolates were strongly positive similar to Mathur et al., (2006) (11.8\%) and slightly lower than by above authors (Table 1 and Fig. 1).

Table.1 Detection of biofilm formation by Staphylococcus aureus using tube method

\begin{tabular}{|l|l|l|l|}
\hline Observation & \multirow{2}{*}{ Inference } & S.aureus & \\
\cline { 3 - 4 } & & Number & Percentage \\
\hline $3+$ & Strong & 07 & $12.28 \%$ \\
\hline $2+$ & Moderate & 29 & $50.88 \%$ \\
\hline $0 / 1$ & Negative & 21 & $36.84 \%$ \\
\hline Total & & 57 & $100 \%$ \\
\hline
\end{tabular}

Out of the 57 isolates tested for biofilm by tube method, 07 (12.28\%) were strongly positive. However maximum number of isolates $29(50.88 \%)$ were moderately positive and 21 (36.84\%) did not show any biofilm formation. 
Table.2 Detection of biofilm formation by Staphylococcus aureus using tissue culture plate method

\begin{tabular}{|l|l|l|l|}
\hline Observation & Inference & \multicolumn{2}{|l|}{ S.aureus } \\
\cline { 3 - 4 } OD $_{\mathbf{5 7 0}}$ & & Number & Percentage \\
\hline$>0.240$ & Strong & 08 & $14.04 \%$ \\
\hline $0.120-0.240$ & Moderate & 29 & $50.88 \%$ \\
\hline$<0.120$ & Negative & 20 & $35.09 \%$ \\
\hline Total & & 57 & $100 \%$ \\
\hline
\end{tabular}

In S.aureus by tissue culture plate method, 8 (14.04\%) isolates were strongly positive for biofilm production, $29(50.88 \%)$ were moderate biofilm producers whereas $20(35.09 \%)$ were negative for biofilm formation

Table.3 Comparison of results from TM and TCP tests on S. aureus, using TCP as the gold standard

\begin{tabular}{|c|c|c|c|c|}
\hline S.aureus & \multicolumn{4}{|l|}{ Tissue Culture Plate Method } \\
\hline Tube Method & Positive & Negative & Total & $\%$ \\
\hline Positive & 36 & 00 & 36 & 63.16 \\
\hline Negative & 01 & 20 & 21 & 36.84 \\
\hline Total & 37 & 20 & 57 & 100 \\
\hline
\end{tabular}

Sensitivity $-97.30 \%$

Specificity $-100 \%$

Positive Predictive Value (PPV) - 100\%

Negative Predictive Value (NPV) - 95.24\%

Kappa -0.96

When the results of TM method for biofilm formation was compared with TCP, it was found that the specificity and sensitivity was $100 \%$ and $97.30 \%$.

Fig.1 Tube method for detection of biofilm formation

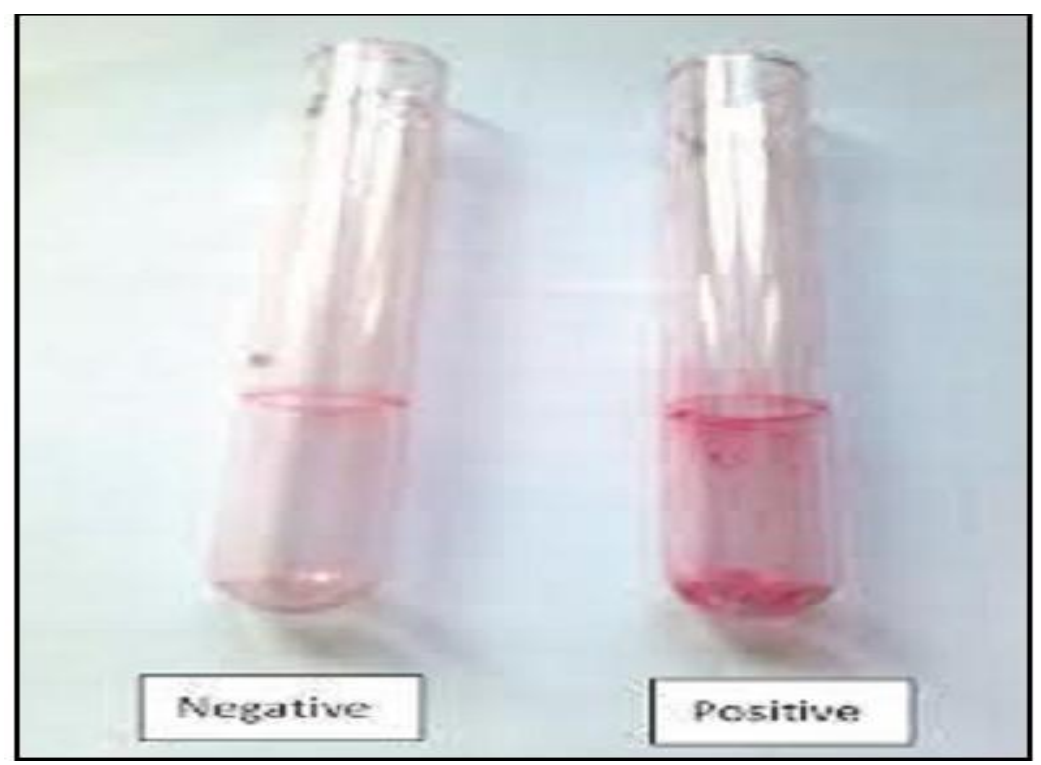

In the study by Ammendolia et al., (1999) who detected biofilm formation using Tisse Culture Plate method, a high percentage of
S.aureus strains (88.9\%) were found to produce slime/biofilm as deected by plate test. In the study by Fatima et al., (2011) of the 
total of 262 isolates of s.aureus, 38(14.5\%) were strongly positive, and $132(50.38 \%)$ were moderate positive.

The present study findings in S.aureus; 8 $(14.04 \%)$ isolates were strongly positive for biofilm production is similar to Christensen et al., (1982) but moderate biofilm producers of $29(50.88 \%)$ was higher, Negative biofilm producers $20(35.09 \%)$ were lower.

The present study findings were similar to the above authors. In the study by Fatima et al., (2011) the comparision between tube method and tissue culture plate method showed a sensitivity of $95.78 \%$, specificity of $99.49 \%$, a PPV of $99.11 \%$. and a NPV of $95.29 \%$ (Tables 2 and 3 ).

In the present study for the similar comparision between methods sensitivity was $97.30 \%$, specificity of $100 \%$, PPV of $100 \%$ and NPV of $95.24 \%$. Findings in our study were similar to the above studies.

In conclusion, as biofilm formation has an important role in pathogenicity of infections, its detection should be mandatory in a laboratory set up. Since tube method is more qualitative and reliable method to detect biofilm producing organism, it can be used as a general screening method for detection of biofilm producing bacteria in laboratories. TCP method is an accurate and reproducible screening method for biofilm production. All isolates can be tested by two methods, which will definitely improve the sensitivity for biofilm detection.

\section{References}

Ammendiola, M.G., Rosa, R.D., Montanaro, L.M., Aricola, C.R., Baldassarri, L. 1999. Slime production and expression of the slime associated antigens and expression of the slime associated antigens by Staphylococcal clinical isolates. J. Clin. Microbiol., 37(10): 3235-3238.

Christensen, G.D., Bisno, A.L., Simpsom, W.A., Beachey, E.H. 1982. Adherence of slime producing strains of Staphylococcus epidermidis to smooth surfaces. Infect Immun., 37: 318-326.

Fatima, K., Indu, S., Meher, R., Tariq, M., Sharma, S.C. 2011. Detection of biofilm formation in Staphylococcus aureus. Does it have a role in treatment of MRSA infections? Trends in Med. Res., 6(2): 116-123.

Freeman, D.J., Falkner, F.R., Keane, C.T. 1989. New method for detecting slime production by coagulase-negative Staphylococci. J. Clin. Pathol., $\quad$ 42: 872-874. doi:10.1136/jcp.42.8.872

Mathur, T., Singhal, S., Khan, S., Upadhyay, D.J., Fatma, T., Rattan, A. 2006. Detection of biofilm formation among the clinical isolates of Staphylococci: an evaluation of three different screening methods. Indian J. Med. Microbiol., 24(1): 25-9.

Oliveira, A., Lourdes, M., Cunha, R.S. 2010. Comparison of methods for detection of biofilm production in coagulase-negative Staphylococci. BMC Res Notes, 3: 260.

Robert, D.W., Kamila, O., Malgorzata, K., Danuta, I., Arkadiusz, D., Tomaz, M., Michal, K., Maria, M., Tomasz, J.W. 2014. Biofilm formation and Antimicrobial Susceptibility of Staphylococcus epidermidis Strains from a hospital Environment. Int. J. Environ. Res. Publich Health, 11: 4619-4633.

\section{How to cite this article:}

Ram Mohan Reddy, K. 2017. Tube Adherence Test as a Screening Tool for Detection of Biofilm Formation among Staphylococcus aureus. Int.J.Curr.Microbiol.App.Sci. 6(8): 13251329. doi: https://doi.org/10.20546/ijcmas.2017.608.161 